\title{
The supporting role of the midwife during the first 14 days of breastfeeding: A descriptive qualitative study in maternity wards and primary healthcare
}

\begin{abstract}
Introduction

In Flanders, Belgium, $78.2 \%$ of the women exclusively breastfeed their babies following birth. However, after three months just one third of those babies is breastfed exclusively. This is in contrast with the WHO recommendations of a minimum of six months. Studies reveal that women are not always satisfied with the breastfeeding support they receive from their midwives, which can lead to early cessation of breastfeeding. The general aim of this study is to gain an in-depth understanding of the supporting role of midwives in breastfeeding support during the first two weeks after birth and compare it to the needs of the breastfeeding women.

\section{Methods}

A qualitative approach was used with an observational design and a focus group study. Two researchers performed non-participative observations in both hospital and primary healthcare settings. Midwife-mother interactions during breastfeeding support (midwives, $n=21$; mothers, $n=59$ ) were observed. Analysis of the data gathered from two focus groups with midwives $(n=10)$ and two focus groups with mothers $(n=9)$ provides an in-depth understanding of the supporting role of midwives during breastfeeding and helps to investigate whether that role corresponds to the mothers' needs both from the mothers' and the midwives' perspective. Observations and focus groups were analyzed using thematic analysis.
\end{abstract}

\section{Findings}

The analysis showed that both midwives and mothers want the breastfeeding to be a success. Generally, the production of breastmilk is a priority, for both the midwives and the mothers. Overall a hands-on approach regarding breastfeeding support was observed, mainly in the hospital settings when compared with the findings from the primary healthcare settings. A hands-on approach often occurs without asking the mother's consent.

During the breastfeeding support, a professional relationship between the midwife and the mother is fostered. In primary healthcare there is one - to - one support which is contrary to hospital care, resulting in a personalized relationship. Breastfeeding support is influenced by personal (e.g. negative belief in breastfeeding policies) and external context factors (e.g. time restraints).

\section{Conclusion}

The research data revealed three topics on breastfeeding support by the midwife which were the focus, the approach and the relationship between the midwife and the mothers. The way the midwife 
provides this support varies due to the setting (hospital or primary health care) and due to personal convictions of midwives and mothers. The study shows the importance of providing breastfeeding support which is tailored to individual needs of the mother.

\section{Introduction}

Breastfeeding is widely recognized as the best option for infant feeding and is considered a critical element for public health (American Association of Pediatrics, 2012; Lawrence, 2012). The health benefits of breastfeeding for children and mothers have been well documented (Bagci Bosi et al., 2016; Binns et al., 2016; Gertosio et al., 2016; Renfrew et al., 2012). The World Health Organisation (WHO) recommends that women should breastfeed exclusively for at least six months. After six months, breastfeeding should continue as an important component of the infant's nutrition for up to two years (World Health Organization, 2016).

Globally breastfeeding rates and in particular exclusive breastfeeding rates remain low (Schmied et al., 2011; Burns et al., 2012). In the Flemish Northern part of Belgium, $78,2 \%$ of the women exclusively breastfeed their babies following birth. The Flemish agency "Public Health, Welfare and Family" reports that on day six breastfeeding rates decrease to $65.3 \%$ and at three months only $33.4 \%$ of the babies are breastfed exclusively (Kind \& Gezin, 2017). Across European region member states, after six months the prevalence of exclusive breastfeeding declines to a minimum of $1 \%$ (Greece, Finland and UK) and a maximum of $49 \%$ (Slovakia) with a mean of 13\% (Bagci Bosi et al.,2016).

The importance of breastfeeding support is well documented in international research (McFadden et al., 2017; Swerts et al., 2016; Zhang et al., 2018). Mothers identify adequate breastfeeding knowledge and a positive attitude towards breastfeeding as important success factors for exclusive breastfeeding at 4 months (Zhang et al., 2018). A common reason for breastfeeding cessation is often caused by, the wrong perceptions of the mother, of inadequate milk supply during the first weeks after birth (Zhang et al., 2018). This perception interferes with exclusive breastfeeding rates and can be intercepted through education (Gross et al., 2011; Zhang et al., 2018). A systematic review from our team, investigating the role of the midwife in breastfeeding support found that midwives value breastfeeding education and breastfeeding support as a significant part of their role as a midwife (Swerts et al., 2016). The meta synthesis from Schmied et al. (2011) revealed that women are not always satisfied with the breastfeeding support they receive from their midwives during the first weeks after birth. Dissatisfaction is particularly seen in the hospital setting and less in the primary healthcare setting, possibly because of fragmented care, and can lead to early cessation of breastfeeding. McFadden et al. (2017) stated in a systematic literature review that when midwives offer breastfeeding support, the 
duration and exclusivity of breastfeeding will increase and both the quantity and the quality have an impact on early cessation (Mc Fadden, 2017).

Swerts et al. (2016) describe two perspectives that midwives use to support the breastfeeding woman: 'the midwife as technical expert' and 'the midwife as skilled companion'. The 'technical expert' midwife is mainly breast centred, focuses on techniques, uses the hands-on approach and sees the woman as a novice. The 'skilled companion' midwife is woman centred, focuses on the mother - infant relationship and uses a hands-off approach during the breastfeeding support. Midwives working in a hospital setting face many barriers when providing breastfeeding support, such as time shortage, which makes it difficult for them to carry out their preferred role as a 'skilled companion'. These barriers can influence the breastfeeding support negatively. Supporting factors, for example implementing evidence based breastfeeding guidelines, have a positive influence on the breastfeeding support (Swerts et al., 2016).

\section{Aim}

The general aim of this study is to gain an in-depth description of the role of the midwife in the provision of breastfeeding support during the first two weeks after birth, in hospital and in the primary healthcare setting, and to compare it to the needs of the breastfeeding women by field observations. Additionally, focus groups were used to explore the supporting and restricting factors that midwives experience and the expectations and experiences of the women towards the midwife during breastfeeding support.

\section{Methods}

\section{Design}

A qualitative approach was used with an observational design and a focus group study.

In the first part of the study, two researchers performed non-participative observations during breastfeeding support from midwives. The aim was to observe the midwife in her natural setting with focus on the interaction between her and the breastfeeding women. These observations allowed the researchers to systematically describe and analyze the interventions and attitudes of midwives regarding breastfeeding support (Mortelmans, 2013). During these non - participative observations, the researchers did not intervene at any moment during the care of the midwife. Midwives were informed about the general study aim, the role of the midwife during breastfeeding support, but not on any specific focus to avoid that this would cause them to behave differently during the observations. These observations took place during the first 14 days postnatal, with the exception of the first feeding. 
In the second part, midwives and breastfeeding women who participated in the observational part of the study were invited to participate in focus groups. Focus groups were suitable to further question the midwives and the mothers about the breastfeeding support (Mortelmans, 2013). The focus groups with midwives explored the supporting and restricting factors that they experienced during their breastfeeding support and their role as a midwife concerning breastfeeding support. The focus groups with the mothers inquired into their expectations and experiences towards their midwives regarding breastfeeding support. Because of the low response rate the researchers invited additional midwives and women who did not participate in the observational study.

The format for the focus groups was based on a questionnaire that contained a topic list with openended questions. The questionnaire was developed by the researchers based on a systematic review regarding the role of the midwife in supporting breastfeeding women (Swerts et al., 2016).

Multiple data-collection methods (observations and focus-groups) in this research allowed different perspectives on breastfeeding support provided by the midwife to be captured (Mortelmans, 2013).

\section{Ethics}

The Medical Ethics Committees of both hospital sites gave formal approval for this study (B371201524536). All midwives and mothers that participated in the study provided written informed consent.

\section{Setting}

The observational part of this study was performed in both hospital and primary healthcare (home care) settings. Observations in the hospital setting occurred in two regional hospital sites. Both hospitals were publicly funded and had a maternity ward with close to 2000 births per year. Hospital 1 was BFHI (Baby Friendly Hospital Initiative) labelled, while hospital 2 was not. The observations in the primary healthcare settings were performed with midwives employed by a primary healthcare organization (site 3) and with self-employed midwives (site 4) who were not affiliated with a primary health care organization. Focus groups were organised in a neutral setting of the University College where the researchers were working.

\section{Participants}

\section{Midwives}

For the observational part of this study a purposive sampling was used. Midwives from all settings were informed about the study and asked to willingly participate. The midwives working at the two hospital sites received verbal information during staff meetings about the observational study and the focus group study, and a written information document about the study. Midwives working in the 
primary healthcare organization (site 3 ) were contacted through the responsible midwife and also received written information about the study and a letter requesting for their participation in the study. The self-employed midwives (site 4) were contacted through the address list of the Flemish Organisation of Midwives (VBOV, vzw). For practical reasons, participants were mainly recruited in the provinces of Limburg and Antwerp, in the Flemish Northern part of Belgium. Participation was voluntary. Midwives who wanted to participate were contacted and invited to sign an informed consent. In order to improve the response rate, the researchers visited the hospital regularly to contact the midwives in-person and ask if they were interested in participating in the study.

A total of 159 midwives from both the hospital and primary healthcare settings were invited to participate in the observational study. Of the 159 midwives, 21 agreed to participate (13.21\%) and they were also invited (verbally and by email) to participate in a focus group. Twelve midwives agreed to participate in the focus group, due to the low response rate the researchers invited other midwives who did not participate in the observational study. Another 44 midwives working in primary healthcare settings were invited by email and nine leading midwives were contacted to ask if midwives from their team wanted to participate. Eight of these midwives (team members) confirmed their participation. A total of 20 midwives finally agreed to participate the focus groups therefore two focus groups were organized. Four midwives did not attend for the first focus group and seven midwives did not attend the second focus group. Finally, ten midwives participated the focus group. After discussing the preliminary results from the thematic analysis, the research group agreed that data saturation had been reached. Therefore no additional focus groups were organized.

\section{Women}

On the hospital ward and in the primary healthcare setting, all women whose midwives were participating in the observational part of the study, were invited to participate if they could understand written and verbal Dutch (Dutch is spoken in the Northern part of Belgium) and were currently breastfeeding. Mothers who were hospitalized on the maternal intensive care ward and/or had babies that were hospitalized on the neonatal intensive care ward were excluded.

The midwives asked the mothers if they wanted to participate in the observational part of the study. When they were interested in participating the researchers gave further information (verbal information and a written document) about the study. It was stated clearly that participation or nonparticipation in this study did not influence the support provided by the midwife. If the mothers agreed to participate, they signed an informed consent. Women were also informed that they could stop their participation at any point in time. After the observations the women were invited by the researcher to participate in a focus group. 
59 women participated in the observational study with only three participating in the focus group. The main cited reason for not to participating was 'being too busy'. Therefore, the researchers invited other women through social media. To participate they had to understand written and verbal Dutch. Ten women finally agreed to participate which eventuated in nine of them attending.

\section{Demographic details from the participants (table 1)}

Observations of breastfeeding support by the midwife

21 midwives participated in the observational part of the study, 14 (67\%) worked in a hospital setting and seven (33\%) worked as midwives in the primary healthcare setting. Characteristics of the midwives are shown in table 1.

A total of 59 women were under the care of these midwives during the observation period with all 59 women giving permission to participate in the study. Of the 59 women, 46 women (78\%) were observed at the hospital and 13 women (22\%) were observed at the primary healthcare setting. With these 59 women, a total of 63 care interactions were observed. The mothers had a mean age of 30.5 years (Range $24-40)$, and $42 \%(n=25)$ were primiparae and $58 \%(n=34)$ were multiparae.

Focus groups with midwives

We organized two focus groups with the midwives (total $n=10$ ). The mean age of the midwives was 39 years (Range $30-63$ ). They had a mean experience of 15.7 years (Range $9-28$ ). All of the midwives that contributed to the focus groups had a bachelor's degree in midwifery $(n=10)$ and seven of them had a degree in lactation consultancy. All, except one midwife breastfed their own children.

\section{Focus groups with women}

We organized two focus groups with mothers $(n=9)$. The mean age of the women in the focus group was 29.5 years (Range $25-36$ ), and $33 \%(n=3)$ were primiparae and $67 \%(n=6)$ multiparae. In the first focus group not a single primipara participated, therefore a second focus group was organised. To meet saturation criteria, the researchers recruited primiparous women through social media where three primiparae agreed to participate. All women, except one gave birth in the hospital. Of the nine women, eight of them received breastfeeding support at home by a midwife working in primary healthcare.

Table 1 Demographic details from the participants 


\begin{tabular}{|l|l|l|}
\hline MIDWIVES & OBSERVATIONS (N=21) & FOCUSGROUPS (N=10) \\
\hline Mean age (years, range) & $31(24-63)$ & $39(30-63)$ \\
\hline Mean experience (years, range) & $11(1-28)$ & $15,7(9-28)$ \\
\hline Bachelor's degree (\%) & $100 \%$ & $100 \%$ \\
\hline Lactation consultancy & $38 \%$ & $70 \%$ \\
\hline Master's degree & $9,5 \%$ & $0 \%$ \\
\hline Breastfed at least one own child & $81 \%$ & $90 \%$ \\
\hline
\end{tabular}

\begin{tabular}{|l|l|l|}
\hline WOMEN & OBSERVATIONS (N=59) & FOCUSGROUPS (N=9) \\
\hline Mean age (years, range) & $30.5(24-40)$ & $29.5(25-36)$ \\
\hline Hospital setting $(\mathrm{N}, \%)$ & $46(78 \%)$ & $\begin{array}{l}8(89 \%) \text { Hospital birth + follow } \\
\text { up primary healthcare setting }\end{array}$ \\
\hline Primary healthcare setting & $13(22 \%)$ & $1(11 \%)$ Home birth \\
\hline Primiparae & $25(42 \%)$ & $3(33 \%)$ \\
\hline Multiparae & $34(58 \%)$ & $6(67 \%)$ \\
\hline
\end{tabular}

\section{Data-collection}

\section{Observations}

A total of 63 care interactions were observed. Observations were described on observation sheets and were completed with the field notes of the researchers. During the observations, the researcher did not take notes in order not to interrupt the midwife. After the observation the field notes were written down on an observation sheet and/or observations were verbally recorded using a voice recorder. Together with the field notes, the researchers also wrote down the methodological observations that possibly could bias the data (for example the presence of visitors in the room) (Mortelmans, 2013).

\section{Focus groups}

Four focus groups were organised, two with midwives and two with mothers. The focus groups were digitally recorded on a voice recorder and verbatim transcriptions were made by the two primary researchers who attended the focus groups (MS and EW). By using this kind of transcription, everything said by both participants and the researcher leading the focus group was captured, which increases the credibility of the analysis (Morrison and James, 2009, Hennink et al., 2011b).

The first focus group with the mothers was led by one researcher (JV) and observed by the other two researchers (MS and EW) who also took notes and recorded the focus groups. The second focus group was led by one researcher (MS) and observed by EW.

\section{Data analysis}


Thematic analysis is described as an appropriate method to explore experiences, meanings, and the reality of participants (Braun and Clarke, 2006). This method of analysis is not related to one fixed theoretical background and can thus be used within different contexts and for different purposes (Braun and Clarke, 2006; Kohler Riessman, 2008). Given the aim of this study is to explore the actual roles of the midwives, their experiences and the experiences of breastfeeding women, inductive thematic analysis was found to be the most suitable method to analyze the data (observation sheets and field notes) derived from both the observations and the focus groups.

Data analysis was performed following the steps for thematic analysis as described by Braun and Clarke (2006), to identify and report themes. We reviewed and refined the coding process until we had a thematic map that we were satisfied with. Furthermore, we defined and further refined the themes in order to grasp the "essence" of what each theme is about. We went back to collated data extracts for each theme, and organized them into a coherent account, with accompanying narratives.

To ensure that participants' anonymity during analysis of both the observations and the focus groups was guaranteed, any identifying information was removed and replaced with descriptors (Hennink et al., 2011b).

To increase the trustworthiness of the study, $\mathrm{JL}$ who was not involved in the initial coding, reviewed these themes and the data and added her interpretations to refine the analyses of the observations and the focus groups (Mays and Pope, 1995). The level of agreement between all researchers was assessed and discrepancies in coding were discussed. Furthermore, the findings of the observations and the key issues of the focus groups were checked (member check) with the participants during and at the end of the focus groups. At that moment, all participants were given the opportunity to add additional information if needed or to correct the researchers (Kvale, 1996).

\section{Saturation}

Data was collected between January 2016 and September 2016. Inclusion of participants continued until data saturation was reached, which means that observations were stopped when no new information was being obtained from additional observations (Hennink et al., 2011a). After discussing the preliminary results from the thematic analysis, the research group agreed unanimously that data saturation had been reached.

\section{Findings}

\section{Breastfeeding support from midwives}


The thematic analysis of the data revealed three important topics on breastfeeding support provided by the midwife: the focus of the midwife during breastfeeding support, the approach of the midwife (and expectations from both midwife and woman) and the relationship between the midwife and the woman during the breastfeeding support in the first two weeks after birth.

\section{FOCUS of the midwife}

The results showed that both the midwives and the mothers want the breastfeeding to be successful. The production of breastmilk and the latch is priority for both parties, especially during the first days after birth. This was seen during the observations and confirmed by both the midwives and the mothers during the focus groups.

Midwives (in hospital setting and in primary healthcare) indicate that they find it important to respond to the individual needs and the questions of the mother at that specific moment. This was confirmed by the mothers in the focus groups. For example, mothers asked the midwives many questions about the production and the frequency of breastfeeding. According to the midwives, this is the reason why the production of breastmilk is often the focus of the conversation. Mothers indicate that they are regularly uncertain about their production and therefore have a great need for information on this topic. In the hospital setting the babies are weighed daily. This leads to more stressed mothers who are struggling with breastfeeding because this emphasizes even more the recovery or weight loss which is related to a (un)successful production of breastmilk. This is less observed in primary healthcare. Midwives indicated during the focus groups that daily weighing causes the mother's stress and negatively affects the production of breastmilk. They also stated (in hospital setting and in primary healthcare) that when they feel that the mother experiences too much stress, they tried not to focus on the production. This was also identified during the observations.

"you don't need a scale to know that your child has enough" - Field note $\mathrm{nr} .37$ (midwife - hospital setting)

The wellbeing of the mother is also important for the midwife. While supporting the breastfeeding mother, the midwife asks about the wellbeing and the motivation of the mother to breastfeed. Mothers indicated that they found this very important. It is important that the mothers remain intrinsically motivated to continue breastfeeding through midwives asking what the mother needs when certain problems arise and identifying solutions.

"you're doing a great job but you have to feel good about it" - Field note nr. 29 (midwife - hospital setting)

Midwives stated during the focus groups that they sometimes find it frustrating that breastfeeding is not always a priority for the mothers who seem to be occupied with other things, for example, being 
constantly busy with their smartphone or when people come to visit. Positive support from their partner, family and friends is related to the motivation of the mother to continue breastfeeding.

\section{APPROACH and EXPECTATIONS}

The observations showed a recurring pattern in the support of the breastfeeding mothers. All midwives used either the hands-on or the hands-off approach to support breastfeeding. They observed the mother and her child during the breastfeeding and they gave information and practical advice to the mother. However, how midwives provide support, differs with the setting and the midwife.

\section{Hands on - hands off}

During breastfeeding support, midwives frequently use hands-on support. This means that the midwife physically touches the breast of the women. The observations showed that this is a common practice in the hospital setting, more than in the primary healthcare setting. The reasons for hands-on support are diverse. For example, manually expressing milk from the breast, manually checking the softness of the breast, the repositioning of the baby on the breast or manually attaching the baby on to the breast. The hands-on support often happens without asking the permission of the mother. This was confirmed by the midwives and the mothers in the focus groups, although some midwives believe that women can feel intuitively from their behavior that they are going to touch the breast.

The mothers stated during the focus groups that they do not mind if the midwife touches the breast, if it is necessary for the breastfeeding to succeed. Nevertheless, they want the midwife to ask their permission or at least warn them before they touch the breast, as they are startled the first time it happens.

"I don't remember anyone ever asking me ... can I touch your breast? ..." - Focus group mothers

On the other hand, observations showed that breastfeeding support can occur with the hands-off approach, meaning without the physical intervention of the midwife. This kind of support is observed in both the hospital and in primary healthcare setting. When using the hands-off approach, the woman independently attached the baby to the breast and the midwife observed and verbally corrected and coached the woman. Some midwives prefer a more visual approach, by showing the position on their own body. The hands-off approach is mostly seen in the primary healthcare setting or when the midwife supports mothers that already have breastfeeding experience (multipara). 
All midwives in the focus groups stated that mothers should get the space and time they need to autonomously attach their baby on the breast, and intervention by the midwife should only occur if the mothers ask for it or if the baby is getting insufficient access to the breast. The midwives acknowledged in the focus groups that in ideal circumstances breastfeeding support should be done by the hands-off approach. Nevertheless, they also recognize that often in the hospital setting, they intervene too quickly with a hands-on approach due to time pressure. They think that intervening with a hands-on approach is less time consuming than the hands-off approach, so that leaves them with more time to fulfill other duties and tasks on the ward. Nevertheless, during the observations it showed that when the ward was not busy, midwives still used the hands-on approach more often.

Midwives from the hospital setting also indicate that the hands-on approach is more often used with mothers who themselves take little initiative to put their babies on the breast and/or are less motivated to breastfeed.

Midwives working in the primary healthcare setting have a different view on the breastfeeding support as they believe that investing time in assisting the mother to independently breastfeed is less time consuming in the end. The reason for such an approach in the primary healthcare setting is that women cannot physically have access to a midwife around the clock or every hour of the day, so preparing the mother for the next 24 hours is essential. Midwives from the primary healthcare setting realize that a private home visit offers more time and therefore more opportunity to invest in handsoff breastfeeding support compared to the midwives of the hospital setting. Some midwives from the hospital setting stated that when they spend more time with the breastfeeding mother, for example, to use the hands-off approach, when the ward is busy, their colleagues do not appreciate that as other duties remain undone. This causes frustrations among midwives.

\section{Observing}

All the midwives in the focus groups stated that observation of the breastfeeding is very important and necessary to give good breastfeeding support. When they cannot fully observe the breastfeeding, it is more difficult to give advice and additional information or detect possible difficulties and address these.

"You're doing very well but i would bring him quicker towards your breast" - Field note nr. 19 (midwife primary healthcare setting)

The observations however showed a big difference between the different settings and between the midwives themselves. In the hospital setting, the observations revealed that due to time pressure and other tasks like answering the phone, the midwife does not stay and observe a full breastfeeding. She 
helps attach the baby if required and then she leaves the room to fulfill other tasks and priorities. She only returns when help is asked for, leading to fragmented care and support. Some midwives seem to overcome these barriers and observe the whole breastfeeding process. Some midwives stated in the focus groups that they are not always satisfied with the choice of their colleague to observe the whole feeding, as she is left alone with all the other duties. The mothers in the focus groups recognize the problem of fragmented care and do not expect that the midwife observes the entire breastfeeding. They acknowledge the fact that it can be very busy. Mothers however appreciate it when the midwife does observe their breastfeeding and can give personal, practical advice. They also confirmed that the midwife is always available when needed, although if a problem occurs during the breastfeeding and the midwife is not present, that they sometimes had to wait a while before the midwife came to see them.

In primary healthcare, the midwives observe the whole breastfeeding moment, and the mothers expect this from their midwives. Mothers say that they get more useful tips and tricks from the midwife when she observes the whole breastfeeding from start to finish. Midwives working in primary healthcare recognize that they have more time and less distractions to observe a whole feeding moment, resulting in the care they provide being less fragmented.

\section{Informing}

Midwives give a lot of information during breastfeeding support and they believe that it is an important part of their role as a midwife to give information that is realistic and correct. Midwives indicate that mothers often get unrealistic and inaccurate information, for example from peers, internet sources etc. This information is usually too optimistic and therefore mothers start breastfeeding with an erroneous misconception of breastfeeding and with the wrong motivation. According to the midwives, this leads to early cessation when the breastfeeding does not go as originally envisaged.

"you shouldn't look at the clock but feed on demand ..." - Field note nr. 6 (midwife - hospital setting)

The given information depends on the questions that the mothers have and are often related to the milk production or on breastfeeding problems such as cracked nipples. Midwives from the primary healthcare setting give the mothers additional information and offer them support that prepares them for the next 24 hours.

Mothers clearly stated during the focus groups that they expect that the midwife has the necessary knowledge to provide accurate and realistic information about breastfeeding, and that she can answer 
all her questions. The mothers also said that they liked it when they received advice from midwives who had work experience. Some mothers indicated that they found it difficult when a student midwife came for breastfeeding advice and support because students themselves have little work experience. Midwives also indicated that their experience as a midwife has an important influence on their approach to breastfeeding support. During their career as a midwife they have grown in the way they provide this support.

Mothers like it when midwives talk about their own breastfeeding experience. "Midwives do share sometimes their own breastfeeding experiences", is what they said during the focus groups. Though they know it is important to consciously uncouple their own experience during the breastfeeding support to the mothers, as also their own experience is not the norm.

"If they are really worried about something, then I sometimes say: I also had that same feeling when I was breastfeeding... that is how I felt ... That they also know that it is not because I am a midwife that it has not happened to me or ... "- Focus group midwives

During the focus groups, all midwives and women agreed that information about breastfeeding should be given in an early prenatal stage. Nevertheless, mothers also felt the need to be informed again after giving birth. When mothers give birth in a hospital setting, further support by the midwife at home is highly valued by the mothers.

\section{Coaching and supporting}

The observation in both the hospital and primary healthcare setting showed that midwives coach and support women while breastfeeding. They empower the mothers, which is helpful to improve the selfconfidence and the belief that they can breastfeed independently. This was confirmed by the mothers during the focus groups.

"Take a good look, you're doing very well, ... but I understand that it is annoying and that it hurts ..."- Field note nr. 36 (midwife - hospital setting)

According to the midwives, the self-confidence of the mother has a great influence on their motivation. Many mothers indicate that they want to "try" to breastfeed, often because they have little selfconfidence. Midwives consider it as an important part of their job to assist and motivate these women to believe in themselves. Some midwives indicated during the focus group that they found it frustrating to support a mother who is not motivated to breastfeed. 
The midwives acknowledge the importance for the mother to make an informed decision when problems occur during breastfeeding. They want to support the woman in making a decision, although the final decision lies with the mother. Midwives with a positive personal breastfeeding experience have a great commitment to breastfeeding, indicating that they sometimes feel frustrated when the mothers want to give up too quickly.

"I notice that I sometimes have less patience ... sometimes I think please ... just do it, keep going ... And I sometimes say that: if I have been able to do that three times, then you can do that too I am $100 \%$ convinced of that" - Focus group midwives

The midwife often feels a conflict between what is good for the mother and what is good for breastfeeding. They respect the choice and the limits of the mother although sometimes fear that mothers will regret their choice for cessation of breastfeeding. That is why the midwives also say that they find it important to inform the mothers about the consequences of their decisions. The mothers in the focus groups expressed the need for support in decision making and respecting their boundaries.

\section{RELATIONSHIP BETWEEN MIDWIFE AND MOTHER}

During breastfeeding support, there is a professional relationship formed between the midwife and the mother. The intensity of this relationship varies depending on the setting, the needs and the expectations of the midwife and the mother.

In the hospital setting care is often fragmented because mothers receive care and support by different midwives in one day, resulting in relationships that are more superficial. This differs from the primary healthcare setting where there is one - to - one support by the midwife. Here we see a more intensive and personal relationship. This was confirmed by the midwives and the mothers in the focus groups.

Some midwives from the primary healthcare setting indicated that they have to guard that the relationship with the mother remains professional, maintaining a certain distance between them. When the breastfeeding support is intense and for a longer period of time, mothers sometimes consider them as friend.

"I knew my midwife from during my pregnancy ... I went to the prenatal classes. It's nice that you know the midwife ... I felt connected with her ..." - Focus group mothers 
In the hospital setting there can also be a more intensive relationship depending on the individual needs of the breastfeeding mother, for example, when the mother needs more support during the breastfeeding (primipara) . Mothers (from the hospital setting) indicate that they do not really expect to have a relationship with the midwives because they only see them for a short period of time. However, they indicate that they find it positive when they see a midwife several times because she knows their situation, and if there is a good relationship they consider this of added value. To ensure a positive understanding, the midwives and the mothers have to take into account each other's needs. During the focus groups the mothers said that they found it more important to get good advice and practical tips regarding breastfeeding, instead of having a good relationship with the midwife. In that matter they sometimes found it positive to have contact with different midwives.

Midwives indicate that the trust can also be strengthened if they introduce themselves by name during the first contact and when they address the mother with her first name. This was confirmed by the mothers. In the hospital setting the midwives often say Mrs. or mama and less the mother's first name in comparison to the midwives in primary care.

\section{Discussion and conclusion}

For this research an observational study and a focus group study was conducted. The data analysis revealed three important topics on breastfeeding support by the midwife: the focus of the midwife during breastfeeding support, the approach of the midwife and the relationship between the midwife and the woman.

The research findings show that both the midwife and the mother want the breastfeeding to be successful with the production of breastmilk and the latch priority (focus) for both. In general, the results of the observations and focus group study show a more technical approach (hands-on) regarding breastfeeding support, mainly in the hospital setting and less in the primary healthcare setting. These findings confirm the results of a systematic literature review which revealed that the majority of the midwives provide breastfeeding support as a technical expert (focus on technique, hands-on approach, breast centered) and the minority of the midwives provide support as a skilled companion (women centered care, hands-off approach). However, the way in which the support is given differs depending on the midwife and the setting (Swerts et al., 2016).

The hands-on support is seen more often in the hospital setting and the hands-off approach in primary healthcare (Swerts et al., 2016). Of the 59 women that participated in the observational part of the study, 46 women (78\%) were observed at the hospital and 13 women (22\%) were observed at the primary healthcare setting. The observations in the hospital setting all took place between the first 
and the fourth day after birth. The breastfeeding support could also be influenced by the time of the observation. During this time period mothers often need more help with breastfeeding (Gross et al., 2011). Midwives however working in the hospital setting indicate that they often intervene too quickly with a hands-on approach, so that they have more time to fulfill other duties or tasks on the ward. Mothers do not mind if the midwife uses the hands-on approach if it is necessary for the breastfeeding to succeed but they want the midwife to ask for permission. This does not always happen.

Of the 59 women, $42 \%(n=25)$ were primipara and $58 \%(n=34)$ were multiparae. We could expect to see more hands-off approach given the fact that there were more multipara, experienced mothers. However during the observations, the hands-on approach was seen more often than the hands-off approach. Schmied et al. (2011) emphasizes the importance of anticipating to the needs of the mother, primi - or multipara, during breastfeeding support.

Many midwives indicate that they want to use the hands-off approach and that they want to observe the breastfeeding more often to anticipate to the needs of the mother, but because of the fragmentation of the care in the hospital setting, that desire remains unfulfilled. Midwives in the hospital setting are under greater time pressure because they have to take care for more mothers simultaneously and they often have other administrative assignments and logistical tasks. However, not all hospital midwives experienced these issues as barriers to use the hands-off approach. One can wonder if this is because they prioritize breastfeeding and are personally motivated to invest time in breastfeeding support or because many hospital midwives often continue to work in the same routine, using the hands-on approach, even if the ward is not busy at that time. The nature of the support given by the midwife also depends on the wider culture of care and the culture of the organization as mentioned in the meta-synthesis from Schmied et al. (2011) and the systematic review from Swerts et al. (2016). In the Western society, the medical system is a technocratic model of medicine. Here the main value is mind - body separation, the individual is separated into components (Davis-Floyd, 2001; Dykes, 2005b). This reflects on the technical approach of the midwife, the hands-on breastfeeding support were the care is more breast-centered then women-centered (Dykes, 2005b; Burns et al., 2012; Swerts et al., 2016).

The mean work experience of the midwives participating in the observational study was 11 years. Mothers indicate that they rather get, and accept advice from a midwife who has work experience in comparison to a student-midwife who has no experience. Mothers expect that their midwife has the necessary knowledge about breastfeeding to provide accurate and realistic information. Midwives also value breastfeeding education as a significant part of their role as midwives (Burns et al., 2012; Burns et al., 2013; Mclelland et al., 2014; Swerts et al., 2016). 
Midwives stated during the focus groups that a misconception of breastfeeding, due to incorrect information, leads to early cessation when breastfeeding does not go as well as expected. According to Zhang et al. (2018) there is a greater chance of exclusive breastfeeding at 4 months when the mother has adequate breastfeeding knowledge.

The observation in both the hospital and primary healthcare setting showed that midwives coach and support women while breastfeeding. They empower the mothers, which is helpful to enhance their self-confidence and the belief that they can breastfeed. This was confirmed by the mothers in the focus groups. Midwives consider it as an important part of their job to assist and motivate these women to believe in themselves. Midwives with a positive personal breastfeeding experience have a great commitment to breastfeeding. This personal breastfeeding experience can influence the breastfeeding support (Swerts et al., 2016).

Mothers also indicate that it is important for the midwife to ask about their well-being and to check whether they are intrinsically motivated to (continue to) breastfeed. In addition, Zhang et al. (2018) stated that a positive attitude of the mother is also important to improve the duration of exclusive breastfeeding.

Mothers have different expectations towards the midwives depending on the setting. Mothers from the hospital setting, stated during the focus groups, that they do not expect to build a relationship with the midwife. They know in advance that they will only see them for a short period of time and have a greater need for information and practical advice even if it has been given by several midwives. In the primary healthcare setting there are different expectations from the mothers. These mothers indicated that they would like to have a relationship with their midwife. Mothers assign a different role for their midwife in a different setting and therefore they have different expectations towards midwives. Schmied et al. (2011) reported that women want to have the opportunity to build a relationship with the midwives. According to our research it depends on the setting. More research is needed.

The findings of this research contribute to a more in-depth understanding of the supporting role of the midwife in the breastfeeding support during the first two weeks after birth and the needs of the breastfeeding women. There are differences in the way the breastfeeding support is given and in the expectations of the breastfeeding mother. The differences in the support depend on the midwife and the setting that she works in. It is important that the midwife adjusts her support to the needs of the individual mother (Swerts et al., 2016). 


\section{Limitations}

Several efforts were undertaken to increase the trustworthiness of the study findings. Firstly, two researchers independently analysed the rough data of observations and focus groups and were supported by two senior researchers (researcher triangulation). Secondly, we obtained a heterogeneous sample of participants, by including primiparous as well as experienced breastfeeding mothers from both hospital and primary healthcare settings. (data triangulation). Data were gathered through observations and through focus groups (method triangulation) (Mortelmans, 2013). Due to the voluntary participation, there is a possible selection bias with only motivated midwives and mothers. It was encouraging to hear the perspective of breastfeeding women through focus groups, but the process of recruiting them required extra effort by the researchers which could implicitly be interpreted as selection bias. In total, 63 care interactions were observed and saturation of data was reached in both settings, even for primary healthcare.

\section{References}

American Association of Pediatrics, 2012. Policy statement. Breastfeeding and the use of human milk. Pediatrics 129, 827-841. http://DOI:10.1542/peds.2011-3552.

Bagci Bosi, A.T., Eriksen, K.G., Sobko, T., Wijnhoven, T., Breda, J., 2016. Breastfeeding practices and policies in WHO European Region Member State. Public Health Nutrition 19(4), 753-764. http://DOI: 10.1542/peds.2011-3552.

Binns, C., Lee, M., Low, W, 2016. The Long-Term Public Health Benefits of Breastfeeding. Asia Pacific Yournal of Public Health 28 (1), 7 - 14. https://doi.org/10.1177/1010539515624964

Braun, V., Clarke, V., 2006. Using thematic analysis in psychology. Qualitative Research in Psychology 3, 77-101. http://DOI:10.3402/qhw.v9.26152.

Burns, E., Schmied, V., Fenwick J., Sheehan, A., 2012. Liquid gold from the milkbar: Constructions of breastmilk and breastfeeding women in the language and practices of midwives. Social Science \& Medicine 75, 1737 - 1745. http://DOI:10.1016/j.socscimed.2012.07.035.

Burns, E., Fenwick, J., Sheehan, A., Schmied, V., 2013. Mining for liquid gold: midwifery language and practices associated with early breastfeeding support. Maternal and Child Nutrition 9, $57-73$. 
Davis-Floyd, R., 2001. The technocratic, humanistic, and holistic paradigms of childbirth. International Journal of Gynecology and Obstetrics 75, S5 - S23.

Dykes, F., 2005b. 'Supply' and 'demand': breastfeeding as labour. Social Science and Medicine 60, 2283 $-2293$.

Gertosio, C., Meazza, C., Pagani, S., Bozzola, M., 2016. Breastfeeding and its gamut of benefits. Minerva Pediatrica 68 (3), $201-2012$.

Gross, S., Resnik, A., Nanda, J., Cross - Barnet, C., Augustyn, M., Kelly, L., Paige, D., 2011. Early postpartum: A Critical Period in Setting the Path for Breastfeeding Success. Breastfeeding medicine 6, 407 - 412. http://DOI:10.1089/bfm.2010.0089

Hennink, M., Hutter, I., Bailey, A., 2011a. Qualitative Research Methods. London: Sage Publications Ltd. pp. 83-107.

Hennink, M., Hutter, I., Bailey, A., 2011b. Qualitative Research Methods. London: Sage Publications Ltd. pp. 203-237.

Kind en Gezin, 2017. Kind in Vlaanderen. https://www.kindengezin.be/cijfers-enrapporten/cijfers/borst-en-flesvoeding/vlaams-brussels-en-provinciaal-niveau/default.jsp

Kohler Riessman, C., 2008. Thematic analysis. In: Kohler Riessman, C., editor. Narrative methods for the human sciences. Thousand Oaks, California: Sage Publications Inc. pp. 53-76.

Kvale, S., 1996. InterViews. An introduction to qualitative research interviewing. Thousand Oaks, California: Sage Publications Inc. pp. 187-209.

Lawrence, R., 2012. Breastfeeding - a public health issue, not just a matter of choice. Breastfeed Med 7, 67-68. http://DOI:10.1089/bfm.2012.9990

Mays, N., Pope, C., 1995. Rigour and qualitative research. British Medical Journal 311, 109-112.

McFadden, A., Gavine, A., Renfrew, MJ., Wade, A., Buchanan, P., Taylor, JL., Veitch, E., Rennie, AM., Crowther, SA., Neiman, S., MacGillivray, S., 2017. Support for healthy breastfeeding mothers with 
healthy term babies. Cochrane Database of Systematic Reviews 2017, Issue 2. Art. No.: CD001141. http://DOI:10.1002/14651858.CD001141.pub5.

Mclelland, G., Hall, H., Gilmour, C., Cant, R., 2014. Support needs of breast - feeding women: Views of Australian midwives and health nurses. Midwifery 31, e1 - e6.

Morrison, M., James, S., 2009. Portuguese immigrant families: the impact of acculturation. Family Process 48(1), 151-166.

Mortelmans, D., 2013. Handboek kwalitatieve onderzoeksmethoden. Acco, Leuven.

Renfrew, MJ., McCormick, FM., Wade, A., Quin, B., Dowswell, T., 2012. Support for healthy breastfeeding mothers with healthy term babies. Cochrane Database of Systematic Reviews. Issue 5, 1 -25. http://DOI: 10.1002/14651858.CD001141.pub4.

Schmied, V., Beake S., Sheehand, A., McCourt, C., Dykes, F., 2011. Women's Perceptions and Experiences of Breastfeeding Support: A Metasynthesis. Birth 38, 49 - 60. http://DOI:10.1111/j.1523536X.2010.00446.x.

Swerts, M., Westhof, E., Bogaerts, A., Lemiengre, J., 2016. Supporting breast - feeding women from the perspective of the midwife: A systematic review of the literature. Midwifery $37,32-40$. http://DOI:10.1016/j.midw.2016.02.016.

World Health Organization, 2016. Exclusive breastfeeding. Consulted on August 22 ${ }^{\text {nd }}, 2016$ through http://www.who.int/nutrition/topics/exclusive_breastfeeding/en/.

Zhang, Z., Zhang, L., Wan, H., 2018. What factors influence exclusive breastfeeding based on the theory of planned behaviour. Midwifery 62, 177-182. http://DOI:10.1016/j.midw.2018.04.006. 